NASA Technical Memorandum 102429

AIAA-90-0391

\title{
Efficient Real Gas Navier-Stokes \\ Computations of High Speed \\ Flows Using an LU Scheme
}

William J. Coirier

Lewis Research Center

Cleveland, Ohio

Prepared for the 28th Aerospace Sciences Meeting sponsored by the American Institute of Aeronautics and Astronautics Reno, Nevada, January 8-11, 1990

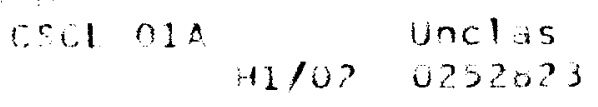




\title{
EFFICIENT REAL GAS NAVIER-STOKES COMPUTATIONS OF HIGH SPEED FLOWS USING AN LU SCHEME
}

\author{
William J. Coirier $\dagger$ \\ NASA Lewis Research Center \\ Cleveland, Ohio 44135
}

\begin{abstract}
An efficient method to account for the chemically frozen thermodynamic and transport properties of air in three dimensional Navier-Stokes calculations has been demonstrated. This approach uses an explicitly specified equation of state (EOS) so that the fluid pressure, temperature and transport properties are directly related to the flow variables. Since the pressure is explicitly known as a general function of the flow variables no assumptions are made regarding the pressure derivatives in the construction of the flux Jacobians. The method is efficient since no sub-iterations are required to deduce the pressure and temperature from the flux variables and allows different equations of state to be easily supplied to the code. The flexibility of the EOS approach is demonstrated by implementing a high order TVD upwinding scheme based upon flux differencing and Van Leer's flux vector splitting. The EOS approach is demonstrated by computing the hypersonic flow through the corner region of two mutually perpendicular flat plates and through a simplified model of a scramjet module gap-seal configuration.
\end{abstract}

\section{Introduction}

The capability to compute chemically frozen single stream flows using an explicitly specified equation of state (hereafter Referred to as EOS) has been added to the PARC3D Navier-Stokes solver. The PARC codes originated as the AIR2D/3D solvers by Pulliam and Steger (Ref. 1), which used the ADI algorithm presented by Beam and Warming (Ref. 2). To increase the computational efficiency and to reduce storage requirements, Pulliam (Ref. 3) diagonalized the block implicit operators of the original Beam-Warming ADI algorithm, resulting in the ARC2D/3D codes. Cooper (Ref. 4) further modified the ARC codes to be used in a production environment for solving the propulsion oriented applications at the Arnold Engineering Development Center, resulting in the PARC codes. Coirier (Ref. 5) replaced the implicit operator with the

$\dagger$ Aerospace Engineer, Computational Methods Br. Member AIAA.

Copyright (C) 1990 by the American Institute of Aeronautics and Astronautics, Inc. No copyright is asserted in the

United States under Title 17, U.S. Code. The U.S. Government has a royalty-free license to exercise all rights under the copyright claimed herein for Governmental purposes.

All other rights are reserved by the copyright owner.
LU-SGS (Lower-Upper Symmetric Gauss-Seidel) algorithm and demonstrated this scheme for hypersonic corner and gap-seal problems. This scheme is based upon an LU decomposition method proposed by Jameson and Turkel (Ref. 6) and demonstrated by Jameson and Yoon for the Euler equations (Ref. 7). In addition, Shuen and Yoon (Ref. 8) and Yu, Tsai and Shuen (Ref. 9) have shown the efficiency and robustness of this algorithm for solving the Navier-Stokes equations fully coupled to the chemical transport equations for computing finite rate chemically reacting flow fields.

For flows where the dissociation and recombination rates are much slower than the fluid residence time (i.e.in the limit as the Damkohler number approaches zero) the fluid can be modelled as a mixture of inert (chemically frozen) gases. When the converse is true (i.e.- the Damkohler number approaches infinity) the fluid can be modelled as if it were in chemical equilibrium. If either of these limitations holds for the flow field to be considered, great simplifications can be made and the resulting computational work reduced. The present research adds the capability to the PARC codes to model general non-mixing chemically frozen flows using an efficient method based upon a generalized equation of state. The flexibility of this approach is demonstrated by implementing a high order TVD upwinding scheme based upon flux differencing (Ref. 10) and Van Leer's flux vector splitting approach (Ref. 11). An equation of state modelling chemically frozen air is then generated and used in the computations of two different types of three dimensional, hypersonic flows: flow through the corner region of two mutually perpendicular flat plates and flow through a simulated scramjet module gap-seal configuration.

\section{Equation of State Formulation}

To account for variable thermodynamic and transport properties it is necessary to relate the pressure, temperature and transport properties to the flow variables. In the majority of flow solvers the fluid specific heats are assumed to be constant, resulting in a simple equation of state, namely: 


$$
\begin{gathered}
p=(\gamma-1) \rho e \\
T=\frac{p}{\rho R} \\
\gamma=\frac{C_{p}}{C_{v}}
\end{gathered}
$$

The speed of sound is then easily found as

$$
\begin{aligned}
a^{2} \equiv\left(\frac{\partial p}{\partial \rho}\right)_{s} & =\frac{\partial p}{\partial \rho}+\frac{p}{\rho^{2}} \frac{\partial p}{\partial e} \\
a^{2} & =\gamma \frac{p}{\rho}
\end{aligned}
$$

For high enthalpy flows the assumption of constant $\gamma$ can lead to over predictions of the temperatures and pressures near stagnation conditions. Real gas effects for compressible flow calculations have been made by many researchers for mixing and non- mixing flows that are considered to be chemically frozen or in chemical equilibrium or non-equilibrium (e.g.- Refs. 8,9,12 and 13). For the frozen and finite rate approaches, once the chemical composition is known (by either solving the species transport equations with or without the species production terms), the pressure and temperature are found in much the same manner. The mixture enthalpy is formed based upon the chemical composition and the individual species enthalpy as

$$
h(T)=\sum_{i=1}^{N} Y_{i} h_{i}
$$

where $h_{i}(T)$ is typically in the form of polynomials in temperature. This is then related to the internal energy (which is readily obtained from the flux variables) by assuming the fluid is ideal, so that

$$
e=h(T)-\frac{p}{\rho}
$$

Then the temperature is found by solving (7) for $\mathrm{T}$ (typically by Newton's iteration or an interval halving method) and the pressure is found from (2). This approach allows mixing or non-mixing chemically frozen flows to be calculated in a finite rate chemistry code by simply switching off the chemical source terms or the species equations completely, but loses efficiency by having to perform the temperature inversion subiterations at each grid point for each global iteration level. Not only is more work required to perform these sub-iterations, the existence of subroutine calls in the do loop structure can devectorize computationally intensive portions of the code (e.g.- pressure terms in the inviscid flux vectors for the residual calculations).
By having an explicit relationship between the pressure, temperature and flux variables, significant savings in work can be achieved by eliminating these subiterations and devectorizing subroutine calls.

Recent work by Shuen, et.al. (Ref. 14) and Liou, et. al (Ref. 15) in developing flux splitting methods for real gases has made use of an explicitly specified equation of state (EOS). This concept was successfully applied by Liou (Ref. 16) for real gas calculations of a corner flow field using a parabolized Navier- Stokes code. By having an explicitly specified EOS, advantages in flexiblity and efficiency for frozen and equilibrium flows are apparent. No "effective $\gamma$ " approximations need to be made in forming flux Jacobians and no sub-iteration procedure is needed to find the pressure and temperature. In addition, the real gas flux splitting methods in Refs. 15 and 16 can be extended to the code once the generalized EOS is operational.

The EOS's generated for this work use thermodynamic and transport data from the NASA Lewis Chemical Equilibrium program, CEC (Ref. 17). CEC is widely used and is accepted as a valuable tool in computing general equilibrium compositions and their properties. This program accesses an extensive data bank that contains thermodynamic data (in the form of polynomials in temperature for individual species specific heats, enthalpy and Gibb's energy) for 741 gaseous species, and transport property data (in the form of polynomials in temperature for individual species molecular viscosities and thermal conductivities) for 154 gaseous species. For mixtures in chemical equilibrium it uses a Gibb's energy minimization procedure that is fully outlined in Ref. 17.

The chemically frozen EOS's generated in this study are made using a least squares surface fit that minimizes the relative error between the fit function and the supplied data. Given the chemical composition, $Y_{i}$, the internal energy and density are computed over a wide range of pressures and temperatures using the thermodynamic data in Reference 17 . Then $p(\rho, e)$ and $T(\rho, e)$ are found by applying the surface fitting procedure. Individual species transport properties are calculated from Ref. 18, from which the mixture properties are found using the Wilke mixing rule. The fits for the mixture laminar viscosity and thermal conductivities are then made from this data set.

Now that $p(\rho, e)$ and $T(\rho, e)$ are known, the derivatives $\frac{\partial p}{\partial \rho}$ and $\frac{\partial p}{\partial e}$ can be easily found. These are used in the formulation of the flux Jacobians in the LU operator and are used in (4) to calculate the speed of sound. By explicitly knowing the pressure derivatives no approximations need to be made in forming the flux Jacobians, such as an "effective gamma" approach. In addition, when forming the inviscid flux vectors the pressure is 
immediately available from an evaluation of the equation of state. The EOS fits are formulated as statement functions used by the code and are contained in FORTRAN include files. By having the EOS's as statement functions, the original vectorized structure of the code is unaffected and no sub-iterations are required to obtain the pressure and temperature. This allows a previously vectorized code to maintain the same basic structure and computational efficiency.

\section{II.a: Chemically Frozen Air Equation of State}

The procedure outlined in the previous section has been used to generate an equation of state for air under the assumption that the fluid is ideal and its composition remains chemically frozen. The mole fraction composition of air is taken to be $X_{N_{2}}=0.78847$ and $X_{O_{2}}=0.21153$, which is used in conjunction with the thermodynamic data from CEC to generate the density and internal energy over an extremely wide pressure and temperature range. The CEC data for enthalpy used to generate the pressure/temperature map has been calculated by statistical mechanics over the temperature range of $200<T($ Kelvin) $<15000$ (Ref. 19). Since the surface fitting procedure used in the generation of the EOS will have the largest errors near the boundaries of the domain, an extremely wide pressure and temperature range was used to compute the density and internal energy. The density and internal energy were computed given the pressure and temperature over the range of $1.0 \times 10^{-4}<P($ Atm $)<100.0$ and $20<T$ (Kelvin) $<15000$. (For $T<200$ (Kelvin) the specific heats are assumed to be equal to their values at $T=200$. Great care was taken in choosing the surface fitting base functions to keep a high level of fitting accuracy over the entire fitting range. The root mean square of the relative error for the pressure, temperature, laminar viscosity and thermal conductivity fits was (respectively) $0.42,0.34,1.7$ and 1.2 percent. Appendix B contains the surface fitted base functions and coefficients for the fits of pressure, temperature, laminar viscosity and thermal conductivities. For comparitive purposes, an EOS for calorically perfect air was also generated following (1), (2) and (5) using $\gamma=1.4$.

Figures 1 and 2 compare the pressures and temperatures for the calorically perfect and thermally perfect EOS's used in the study over a broad temperature and pressure range. Figure 3 compares the laminar viscosities obtained from Sutherland's law, data from Ref. 20 and the present data. Figure 4 compares the thermal conductivities assuming constant Prandtl number $\left(P_{r}=0.72\right), C_{p}$ and Sutherland's law for the viscosity, data from Ref. 20 and the present data. Figure 1 shows the that the differences in pressure between the calorically perfect air and chemically frozen air EOS's are small, but not minor, while Figure 2 shows that the temperature deviations can be quite significant in the higher internal energy ranges. Figures 3 and 4 show that the fitted data follows the NBS standards data more closely than Sutherland's law for both the viscosity and the thermal conductivity.

\section{Numerical Solution Procedure}

The flow solver used in this study solves the Reynolds averaged Navier-Stokes equations cast in divergence form evaluated in a generalized curvilinear coordinate system. The equations are solved implicitly using the LU-SGS algorithm for approximate Newton iteration and are discretized in a finite difference formulation. The original discretization of the convective terms is central differencing with added 1st and 3rd difference dissipation in the manner due to Jameson (Ref. 21). The coefficients controlling the levels of dissipation are kept at their default levels $\left(\kappa_{2}=0.25\right.$ and $\left.\kappa_{4}=.01\right)$ for all of the computations performed in this research. To aid in the shock capturing ability of the code, a second order TVD upwinding scheme based upon flux differencing (Ref. 10) and Van Leer's flux vector splitting (Ref. 11) has been formulated using the generalized equation of state formulation. The following sections briefly describe the implicit and explicit portions of the solution scheme and show how the generalized equation of state is implemented.

\section{III.a: LU-SGS Algorithm}

The LU-SGS (Lower-Upper Symmetric Gauss Seidel) scheme was first demonstrated by Jameson and Yoon (References 22 and 23) in solving the Euler and Navier Stokes equations. For computing flows with finite rate chemistry this scheme has been shown to be very efficient by implicitly solving the Navier-Stokes equations fully coupled to the chemical transport equations (References 8,9 and 24). For solving the Euler or Navier-Stokes equations alone, the resulting scheme is scalar diagonal, and requires only two sweeps with scalar inversions for both two and three dimensional problems. When source terms are included implicitly, the approach results in block diagonal operators. Following the procedure outlined in Reference 5 the hybrid LU-SGS scheme can be derived from the following.

A prototype implicit scheme for solving the NavierStokes equations in delta form may be written as

$$
\left[I+\Delta t\left(D_{\xi} A+D_{\eta} B+D_{\zeta} C\right)\right] \Delta q=-\Delta t R
$$


where $D_{\xi}, D_{\eta}$ and $D_{\zeta}$ are difference operators that approximate $\partial_{\xi}, \partial_{\eta}$ and $\partial_{\zeta}$ and $\mathrm{A}, \mathrm{B}$ and $\mathrm{C}$ are the inviscid flux Jacobians

$$
A=\frac{\partial E}{\partial q}, B=\frac{\partial F}{\partial q}, C=\frac{\partial G}{\partial q}
$$

If the flux Jacobians in (8) are approximated so that they can be split into "+" and "." matrices, where the eigenvalues of the " + " matrices are non-negative and the eigenvalues of the "-" matrices are non-positive, and then differenced according to their sign, (8) may be written as

$$
\begin{gathered}
{\left[I+\Delta t\left(\nabla_{\xi} A^{+}+\Delta_{\xi} A^{-}+\nabla_{\eta} B^{+}+\Delta_{\eta} B^{-}\right.\right.} \\
\left.\left.+\nabla_{\zeta} C^{+}+\Delta_{\zeta} C^{-}\right)\right]=-\Delta t R
\end{gathered}
$$

The choice of conditioning used to form the approximated Jacobians is very important, and following the construction used in Reference 25, the approximated Jacobians are formed as

$$
A^{ \pm}=\frac{A \pm r_{A} I}{2}, B^{ \pm}=\frac{B \pm r_{B} I}{2}, C^{ \pm}=\frac{C \pm r_{C} I}{2}
$$

where

$$
r_{A, B, C}=\beta \max \left(\left|\lambda_{A, B, C}\right|\right)
$$

where $\beta \geq 1.0$ and $\lambda_{A, B, C}$ are the eigenvalues of the flux jacobians. Therefore, using the conditioning in (11), the unfactored scheme may be written as

$$
\begin{gathered}
{\left[I\left(1+\Delta t\left(r_{A}+r_{B}+r_{C}\right)\right)+\Delta t\left(A_{j+1}^{-}+B_{k+1}^{-}+C_{l+1}^{-}\right.\right.} \\
\left.\left.-A_{j-1}^{+}-B_{k-1}^{+}-C_{l-1}^{+}\right)\right] \Delta q=-\Delta t R
\end{gathered}
$$

where $(j, k, l)$ refers to the grid point index. (When an index is omitted it is assumed to be at $\mathbf{j}, \mathrm{k}$ or $\mathrm{l}$ ). The hybrid LU- SGS scheme is then found by factoring (13) into L,D and U operators so that

$$
\begin{gathered}
L D^{-1} U \Delta q=-\Delta t R \\
L=I\left[1+\Delta t\left(r_{A}+r_{B}+r_{C}\right)\right] \\
-\Delta t\left(A_{j-1}^{+}+B_{k-1}^{+}+C_{l-1}^{+}\right) \\
D^{-1}=I\left[1+\Delta t\left(r_{A}+r_{B}+r_{C}\right)\right] \\
U=I\left[1+\Delta t\left(r_{A}+r_{B}+r_{C}\right)\right] \\
+\Delta t\left(A_{j+1}^{-}+B_{k+1}^{-}+C_{l+1}^{-}\right)
\end{gathered}
$$

All of the calculations performed here are only concerned with obtaining the steady state, so an approximate Newton iteration formulation is formed by dividing (13) by $\Delta t$ and taking the limit as $\Delta t$ approaches $\infty$, resulting in

$$
\begin{gathered}
L U \Delta q=-\left(r_{A}+r_{B}+r_{C}\right) R \\
L=\left[I\left(r_{A}+r_{B}+r_{C}\right)-A_{j-1}^{+}-B_{k-1}^{+}-C_{l-1}^{+}\right] \\
U=\left[I\left(r_{A}+r_{B}+r_{C}\right)+A_{j+1}^{-}+B_{k+1}^{-}+C_{l+1}^{-}\right]
\end{gathered}
$$

The solution of $(18)$ is fully vectorizable on planes where $\mathrm{j}+\mathrm{k}+\mathrm{l}=\mathrm{constant}$ and is efficient since it requires only scalar diagonal inversions. The approach is to first solve for an intermediate vector using (19) by sweeping in the direction of planes of increasing $\mathrm{j}+\mathrm{k}+\mathrm{l}$ and then solve for $\Delta q$ using (20) by sweeping in the opposite direction. One advantage of the approximate Newton iteration formulation is that no choice of time stepping or Courant number is required. Results in Reference 5 show that the scheme is slightly more efficient in processing time per iteration than the diagonalized BeamWarming scheme, which was originally in the code.

The inclusion of the generalized equation of state formulation in the LU-SGS scheme is straightforward. Since the pressure derivatives $\frac{\partial p}{\partial \rho}$ and $\frac{\partial p}{\partial e}$ are directly obtainable from the EOS formulation, the inviscid flux Jacobians can be found with no "equivalent gamma" assumptions. Therefore, the only modifications to the LU-SGS scheme using the generalized equation of state formulation is to include the general pressure formulation and its derivatives in the inviscid flux Jacobians, A, B and C in (18), (19) and (20) as well as the speed of sound definition in (4). A general inviscid flux vector including the pressure derivative terms is included in Appendix A.

\section{III.b: Upwinding Approach}

The upwinding approach used in this research is based upon flux differencing (Ref. 10) and Van Leer's flux vector splitting method (Ref. 11). The upwinding approach in Reference 10 uses a monotone interpolation of the non-normalized nodal point, split inviscid fluxes to cell interfaces using a variable order polynomial interpolation scheme. The numerical fluxes evaluated at the cell interfaces consist of the upwinded components, and are written as

$$
\tilde{F}_{i+\frac{1}{2}}=\tilde{F}_{i+\frac{1}{2}}^{+}+\tilde{F}_{i+\frac{1}{2}}^{-}
$$

The interpolation used here interpolates cell face normalized fluxes, simplifying somewhat the approach presented in Reference 10. For the "positive" flux contri- 
butions, the normalized cell interface flux is monotonically interpolated from the nodal values as

$$
\begin{aligned}
& \tilde{F}_{i+\frac{1}{2}}^{+}=F_{i}^{+}+\delta_{i}^{+} \\
& \tilde{F}_{i+\frac{1}{2}}^{-}=F_{i+1}^{-}-\delta_{i+1}^{-}
\end{aligned}
$$

where

$$
\begin{aligned}
& \delta_{i}^{+}=C_{1}^{+} \Delta^{-} F_{i}^{+}+C_{2}^{+} \Delta^{+} F_{i}^{+} \\
& \delta_{i}^{-}=C_{1}^{-} \Delta^{+} F_{i}^{-}+C_{2}^{-} \Delta^{-} F_{i}^{-}
\end{aligned}
$$

The order of the interpolation scheme and its limiting are contained in the coefficients $C_{1,2}^{ \pm}$, which are written as

$$
\begin{aligned}
& C_{1}^{+}=\phi\left(r_{i}^{+}\right)\left[\frac{\theta}{4}\left(1-\kappa-\frac{\sigma}{2}\right)\right] \\
& C_{2}^{+}=\phi\left(\frac{1}{r_{i}^{+}}\right)\left[\frac{\theta}{4}\left(1+\kappa+\frac{\sigma}{2}\right)\right] \\
& C_{1}^{-}=\phi\left(r_{i}^{-}\right)\left[\frac{\theta}{4}\left(1-\kappa+\frac{\sigma}{2}\right)\right] \\
& C_{2}^{-}=\phi\left(\frac{1}{r_{i}^{-}}\right)\left[\frac{\theta}{4}\left(1+\kappa-\frac{\sigma}{2}\right)\right]
\end{aligned}
$$

Following Reference 10 the limiters in (24) are formed based upon the inner products of neighboring flux differences. Although this approach does not strictly adhere to the traditional method of limiting each flux individually, it has been stated to be satisfactory over a sufficiently wide range of conditions. The limiter arguments $r_{i}^{ \pm}$are written as

$$
\begin{aligned}
& r_{i}^{+}=\frac{\Delta^{+} F_{i}^{+} \circ \Delta^{-} F_{i}^{+}}{\Delta^{-} F_{i}^{+} \circ \Delta^{-} F_{i}^{+}} \\
& r_{i}^{-}=\frac{\Delta^{+} F_{i}^{-} \circ \Delta^{-} F_{i}^{-}}{\Delta^{+} F_{i}^{-} \circ \Delta^{+} F_{i}^{-}}
\end{aligned}
$$

The limiter function is taken to be the minimummodulus (min-mod) function, where

$$
\phi(r)=\max (0.0, \min (r, 1.0))
$$

The variables $\kappa, \sigma$ and $\theta$ set the order of the scheme. For all of the following upwinded calculations, 2nd order, full upwinding is used so that $\kappa=-1.0$, $\sigma=0.0$ and $\theta=1.0$. Combining the positive and negative fluxes, the cell face normalized numerical flux is then represented as

$\tilde{F}_{i+\frac{1}{2}}=\frac{1}{2}\left(F_{i}+F_{i+1}\right)-\frac{1}{2} \Delta^{+}\left(F_{i}^{+}-F_{i}^{-}\right)+\left(\delta_{i}^{+}-\delta_{i+1}^{-}\right)$
The first term represents a simple numerical average of the nodal fluxes while the combination of the first and second terms results in a first order upwinding scheme. The third terms represent anti-diffusive fluxes which increase the order of accuracy of the scheme, and are limited to make the scheme monotone.

After the construction of the numerical fluxes at the cell interfaces, the convective contribution to the residual from this coordinate direction is then calculated as

$$
R_{i}=(\tilde{F} \delta)_{i+\frac{1}{2}}-(\tilde{F} \delta)_{i-\frac{1}{2}}
$$

where $\delta$ is the cell interface area. The areas and projected areas at the cell interfaces are found using the transformation metrics evaluated at the nodal points in a consistent manner as (for the $\xi$ - flux)

$$
\begin{aligned}
& \left(\delta_{x}\right)_{i+\frac{1}{2}}=\frac{1}{2}\left[\left(\frac{\xi_{x}}{J}\right)_{i}+\left(\frac{\xi_{x}}{J}\right)_{i+1}\right] \\
& \left(\delta_{y}\right)_{i+\frac{1}{2}}=\frac{1}{2}\left[\left(\frac{\xi_{y}}{J}\right)_{i}+\left(\frac{\xi_{y}}{J}\right)_{i+1}\right] \\
& \left(\delta_{z}\right)_{i+\frac{1}{2}}=\frac{1}{2}\left[\left(\frac{\xi_{z}}{J}\right)_{i}+\left(\frac{\xi_{z}}{J}\right)_{i+1}\right]
\end{aligned}
$$

so that the cell interface area is

$$
\delta=\sqrt{\delta_{x}^{2}+\delta_{y}^{2}+\delta_{z}^{2}}
$$

Since this scheme operates only on flux differences, it is possible to use either a flux vector splitting approach or a flux difference splitting approach. Van Leer's flux vector splitting was chosen due to it's simplicity and efficiency and for the ease in which the generalized equation of state could be included. It should be noted that the equation of state formulation does not preclude the use of Roe's flux differencing approach, which would include the explicitly specified pressure derivatives $\frac{\partial p}{\partial \rho}$ and $\frac{\partial p}{\partial e}$ in the formulation of the transformation matrices. The flux vector splitting used here is formulated including the pressure and sound speed to facilitate using the generalized EOS, so that when $\left|\tilde{M}_{c}\right| \leq 1.0$

$$
F^{ \pm}=\left(\begin{array}{c} 
\pm \frac{\rho a}{4}\left(\tilde{M}_{c} \pm 1\right)^{2} \\
F_{1}^{ \pm}\left(u-\frac{\delta_{z}}{\delta} \frac{P}{\rho a^{2}}\left(\mp 2 a+\tilde{u}_{c}\right)\right) \\
F_{1}^{ \pm}\left(v-\frac{\delta_{y}}{\delta} \frac{P}{\rho a^{2}}\left(\mp 2 a+\tilde{u}_{c}\right)\right) \\
F_{1}^{ \pm}\left(w-\frac{\delta_{z}}{\delta} \frac{P}{\rho a^{2}}\left(\mp 2 a+\tilde{u}_{c}\right)\right) \\
F_{1}^{ \pm}\left[H-m\left(\tilde{u}_{c} \mp a\right)^{2}\right]
\end{array}\right)
$$

and when

$$
\tilde{M}_{c}>1.0: F^{+}=F, F^{-}=0
$$




$$
\tilde{M}_{c}<1.0: F^{+}=0, F^{-}=F
$$

where the contravariant velocity and Mach number are defined as

$$
\begin{aligned}
\tilde{u}_{c} & =\frac{\delta_{x} u+\delta_{y} v+\delta_{z} w}{\delta} \\
\tilde{M}_{c} & =\frac{\tilde{u}_{c}}{a}
\end{aligned}
$$

The parameter $\mathrm{m}$ in $(31)$ is found by requiring the bracketed terms in the energy flux form a perfect square, resulting in

$$
m=\frac{\frac{h}{a^{2}}}{1+2 \frac{h}{a^{2}} \frac{\bar{u}_{c}^{2}}{u^{2}+v^{2}+w^{2}}}
$$

This formulation of $m$ results in an indeterminate expression at stagnation points or along no-slip boundaries, so the relation is modified slightly to give

$$
m=\frac{\frac{h}{a^{2}}}{1+2 \frac{h}{a^{2}}}
$$

The inclusion of the generalized equation of state formulation into this upwinding scheme is straightforward. At each nodal point the flux variables directly yield $\rho$ and $e$ which in turn give $p$ and $a^{2}$ directly from the equation of state from which the flux vector (31) is constructed. This approach using the generalized equation of state requires no special sub-iterations or procedures to find the pressure, sound speed or static enthalpy, and allows the upwinding approach to be fully vectorized. The following sections demonstrate the generalized equation of state approach for a selected number of three dimensional, hypersonic viscous computations.

\section{IV: Corner and Gap Seal Flow Field Study}

Effort is currently underway to investigate the heat transfer and aerodynamic loads in and around a scramjet module gap seal configuration. The seal system must prevent high temperature and pressure gases from leaking through the gaps between the articulating engine panel walls and stationary splitter panels (Ref. 26 ). This system is crucial to the design of hypersonic airbreathing propulsion systems and presents a significant challenge to structural designers to build a viable seal system. To assist the design effort, the present research complements the structural analysis underway in an attempt to better understand the fluid dynamic and heat transfer phenomena in and around the gap seal system. The addition of real gas effects and the inclusion of a high order TVD upwinding scheme are motivated by this effort in the hope that a more thorough understanding of the underlying fluid dynamic processes can help the design effort.

\section{IV.a: Hypersonic Corner Validation}

To obtain some level of confidence in the modelling of the gap seal flow the 3D Navier-Stokes solver has been validated against experimental data taken from a class of flows similar to the gap seal flow (Reference 5). The gap-seal geometry is similar to two mutually perpendicular flat plates (corner) aligned with the freestream flow with a "groove" situated at the plate intersections. Due to this similarity, the 3D code was validated against the experimental data of hypersonic corner flow taken by Cresci (Reference 27). These tests were conducted at a Mach number of 11.8 over a range of Reynolds numbers of $0.15 \times 10^{6}$ to $0.5 \times 10^{6}$ per foot. The data presented in Ref. 5 is axial pressures and heat transfer rates plotted against the hypersonic interaction parameter

$$
\chi=\frac{c^{\frac{1}{2}} M_{\infty}^{3}}{R e_{x}^{\frac{1}{2}}}
$$

for different lateral locations measured from the corner. In addition, surface pressures, heat transfer rates and skin friction data comparisons were made as a function of lateral distance from the corner at various axial locations. This data not only tests the prediction of the mutual interaction of the two plate boundary layers upon each other but also the self induced leading edge pressures caused by the boundary layer growth close to the leading edges.

Comparisons were made against this set of experimental data using the 2nd order upwinding approach outlined here. (The complete set of comparisons using the original central difference formulation are available in Reference 5 . Since the stagnation temperature was low $\left(T_{s t, \infty}=944 K\right)$, the generalized equation of state was not used, so that in essence, this validation effort tests the upwinding approach. A relatively coarse grid (36 X $41 \mathrm{X} 41$ ) was used for plate lengths and widths of 8 and 2.5 inches, respectively. The Reynolds number per foot was taken to be $0.15 \times 10^{6}$, resulting in a Reynolds number based on plate length of $1.0 \times 10^{5}$ from which the flow is assumed to be laminar. The grids were clustered normally in the cross- stream planes using the Robert's transformation with $\beta=1.01$ and axially near the leading edge using $\beta=1.1$, with five grid points extended ahead of the leading edge. 
The comparisons to experiment of the upwinded computation were nearly identical to the central difference results. Since the central difference results already computed in Reference 5 were favorable, only a selected portion of the comparison to experiment are shown here. Figure 5 shows the predicted wall pressures versus the hypersonic interaction parameter at a station 0.125 inches away from the corner intersection, while Figure 6 compares the computed and measured lateral variation of heat transfer at a location of $\chi=8.3$. As can be seen from these figures, the upwinding approach correctly simulates the mutual interaction of the plate boundary layers and the lateral variation of the heat transfer to the plates caused by the leading edge shock and boundary layer interaction.

\section{IV.b: Hypersonic Corner Flow}

The hypersonic laminar flow along the intersection of two mutually perpendicular flat plates at a freestream Mach number of 12 and an altitude of 100,000 feet was calculated using the calorically perfect and thermally perfect air EOS formulations. Both the central difference and 2nd order upwinding approaches were used. The length of the plates is taken to be 12 inches while the width of the plates are both taken to be 2.5 inches. At this altitude the freestream temperature and pressure are 418.79 Rankine and 23.085 psf., resulting in a Reynolds number based on plate length of $1.226 \times 10^{6}$, permitting a laminar calculation. To highlight the real gas effects, adiabatic wall boundary conditions are taken along the plates, while grid line extrapolation is taken at the outflow and lateral boundaries. Although the application of adiabatic wall boundary conditions results in unrealistically high wall temperatures, it is an extreme test of the code robustness and the installation of the real gas effects. Since the thermally perfect EOS formulation allows for a variation in the fluid specific heats while the calorically perfect formulation does not, the difference in wall temperatures between these formulations should be readily apparent. The grid is clustered normally to the walls using a Robert's transformation with $\beta=1.01$ and is clustered axially near the leading edge using $\beta=1.1$. 31 grid points are used in the axial direction on the plates with 5 points extended ahead of the plate leading edges using a grid spacing equal to that of the first cell at the plate leading edge while 41 points are used in the direction normal to each plate.

Figure 7 illustrates the mutual interactions of the plate boundary layers and embedded leading edge shock structure and the downstream growth of the interaction region by showing axial velocity contours in planes perpendicular to the plates at different downstream axial locations. Figure 8 shows the predicted lateral variation of wall temperature approximately 6 inches downstream of the leading edges for the calorically perfect gas equation of state for both the central difference and upwinded discretizations, while Figure 9 shows the chemically frozen air equation of state results. The peak wall temperature locations are practically identical for the two EOS formulations, with the central difference scheme consistently predicting lower peaks than the upwinded scheme. These figures also show that the wall temperature in the noninteractive region computed by the central difference scheme is lower than that computed by the upwind scheme. The non-interactive region of the corner flowfield is far enough downstream of the leading edge so that the leading edge effects of the primary plate are absent, and far enough laterally from the corner so that the opposing plate leading edge shock effects are also absent. This difference in computed non-interactive wall temperatures indicates that the upwinding scheme appears to be less diffusive than the central difference scheme. Figure 9 shows the marked reduction in wall temperature by using the chemically frozen air equation of state, which in essence allows for variation of the specific heats. Similar trends between the differencing schemes as in the calorically perfect computations are also present here.

Differences between results of the two differencing schemes are apparent from a detailed examination of cross stream contours of density and cross stream velocity vector plots. Figure 10 compares contour plots of density from both the central difference and upwinded discretization schemes using the chemically frozen air equation of state. These density contours are taken in a plane perpendicular to the free stream at a distance approximately 3.5 inches from the leading edge of the plates. The central differencing results, shown in Figure 10.a. indicate a merging of the leading edge shock and the plate boundary layer and an oscillatory shock/shock interaction region. The upwinded results, in Figure 10.b, indicate a more clearly defined and separate leading edge shock and boundary layer, and a cleaner shock/shock interaction. These subtle differences between the two discretization schemes produce rather different results in the cross stream velocity field, which is shown in Figure 11. The central difference results, in Figure 11.a, show the beginning of a region of cross stream separation, which results in the downstream development of a weak vortical flow just inboard of the embedded shock. The upwinded results, in Figure 11.b, indicate a much weaker cross stream separation, resulting in a very weak vortical flow downstream. If the upwinding scheme is indeed less dissipative than 
the central difference scheme, as is indicated in the adiabatic wall temperatures, then it is possible that this vortical flow is induced by the numerical dissipation. This finding of numerically induced vortical flow has been previously investigated in detail for conical solutions to the Euler equations for flow over a delta wing (Ref. 28). The results of that study indicated that the existence and strength of the computed cross stream vortical flow on the lee side of a blunt tip delta wing was sensitive to the level of the 2 nd and 4 th order dissipation coefficients. This study indicated that with lower numerical dissipation, the vortical flow disappears. Other computational results have indicated the existence of a vortical structure in corner flow fields, but the findings here indicate that the strength is influenced by the level and type of numerical dissipation. Clearly, more detailed experimental data and more refined numerical experiments are necessary to confidently predict the evolution and behavior of the corner induced vortical flow.

The relative efficiency using the generalized equation of state can be shown by comparing the processing rate per iteration of the different methods. All of the computations were made on a CRAY-YMP using the cft77 compiler. The central difference computations for the calorically perfect formulation and the thermally perfect formulation took $2.4575 \times 10^{-5}$ and $2.7672 \times 10^{-5}$ seconds/iteration/grid point, respectively. This represents a 12.6 percent increase in computing time to include the generalized equation of state. The 2nd order upwinding computations for the calorically perfect and thermally perfect formulations took $3.0829 \times 10^{-5}$ and $3.5453 \times 10^{-5}$ seconds/iteration/grid point, respectively. This represents a 15 percent increase in computing time to include the generalized equation of state. These processing rates also indicate that the addition of the 2nd order upwinding scheme was made at a cost of approximately 25 percent over that of the central differencing scheme.

\section{IV.c: Hypersonic Gap Seal Simulation}

To better understand the heat transfer loads upon the gap seal region in a scramjet combustor it is necessary to have an understanding of the flow field in and above the seal region. This seal prevents hot, high pressure gases from leaking through the juncture of the articulating engine panels and the stationary sideplate walls in a scramjet module (see Ref. 26 for a detailed description of the gap seal methodology). Preliminary work has begun to characterize this flowfield and the resulting heat loads upon the seal region using NavierStokes technology (Ref. 5). Referring to Figure 12, one can see that the basic geometry is similar to two mutually perpendicular flat plates with a "groove" embedded in the corner. Studies show that this groove, or recess, fills up with low momentum and low energy fluid, which reduces the heat loads to the seal. Since this configuration is intended to be used at high Mach numbers, the effects of real gas properties and the inclusion of a high order upwinding scheme may be important in characterizing the heat and aero loads upon the seal.

Simulations of the gap seal flowfield were made at a freestream Mach number of 10 and an altitude of 100,000 feet. The gap seal recess measured 0.25 by 0.25 inches in the corner region of the two mutually perpendicular flat plates. The plates lengths and widths were taken to be 10 and 1 inches, respectively. At this altitude and freestream Mach number, the Reynolds number based on plate length is $8.5139 \times 10^{5}$, permitting a laminar calculation. Grid points were clustered normally to the recess walls and the corner walls and were clustered axially towards the leading edge with 5 points extended ahead of the leading edge. The calculations were performed on a $36 \times 51 \times 51$ grid, of which approximately 78.5 percent of the grid points were active in the calculation. As in the previous case, adiabatic wall boundary conditions were used to highlight the real gas effects. Similar to the corner case, central differencing and 2nd order upwinding were used for both the calorically and thermally perfect equation of state formulations.

Figure 12 shows contours of axial velocity in planes perpendicular to the freestream at different axial locations of the gap seal configuration. This figure shows the rapid thickening of the inter-recess boundary layers due to the over pressures caused by the recess leading edge shocks and their subsequent reflections off of the recess walls. Close inspection of the entrance region in the recess reveals a corner-like flow structure. This similarity to the corner flow only lasts until the interrecess leading edge shocks impinge upon the boundary layers, causing a rapid deceleration of the flow in the recess. The mutual interactions of the recess leading edge shocks and the expulsion of fluid from the recess area results in complicated shock-shock and shock-boundary layer interactions.

Figures 13 and 14 compare cross-stream pressure contours computed with the thermally perfect equation of state for the central difference and upwinding schemes. Figures 13.a and 13.b show pressure contours computed by the central difference and upwinding schemes in a plane located approximately 0.6 inches from the leading edge of the gap seal configuration. This figure shows the sideplate leading edge shock (on the left), the three recess leading edge shocks interaction upon each other and the merging of the cowl in- 
duced leading edge shock and the shock from the right recess wall. The most noticeable difference between the two schemes is the smearing of the cowl leading edge shock to the cowl wall and the oscillations of the pressure near the convex corner of the recess/cowl junction by the central difference approach. Much more noticeable differences are apparent downstream from this plane, as shown in Figures 14.a and 14.b. At this location, the sideplate leading edge shock has traversed out of the recess area and merged with the cowl induced leading edge shock. This results in essentially two separate shock-shock interaction regions: the cowl and sideplate leading edge shock's interactions (the upper interaction region), and the recess leading edge shock's interactions (the interaction still in the recess). Figure 14.a (from the central difference scheme) shows smearing of the cowl leading edge shock and large oscillations that persist near the cowl as well as smaller oscillations inboard of the sideplate induced leading edge shock. Figure 14.b (from the upwinding scheme) has less smearing of the cowl leading edge shock, predicts a more compact cowl/sideplate shock-shock interaction and does not have the severe oscillations near the cowl wall as the central difference scheme's results.

Previous computations using the central difference scheme of the gap seal flow field in Reference 5 indicate a vortical flow on the cowl plate, situated just outboard of the recess. Both the central difference and upwinded computations performed here predict a similar vortical flow, although the strength of this flow is reduced considerably with the upwinded computations. Figures 15.a and 15.b illustrate this by showing cross flow velocity vectors at a location approximately 6 inches from the leading edges of the gap seal configuration. The central difference results, in Figure 15.a, show a much stronger and larger vortical structure than the upwinded results, shown in Figure 15.b. This phenomena is similar to the results in the corner flow simulation, in that although both schemes predict a vortical flow, the central differencing scheme predicted a stronger vortical flow.

Figure 16 compares wall temperatures along the centerline of the recess area for the calorically and thermally perfect equations of state for the upwinding and central difference schemes. Since the thermally perfect equation of state essentially allows for a variation in the fluid specific heats while the calorically perfect equation of state does not, the wall temperature along the centerline is reduced considerably. As in the previous corner case, the relative efficiency using the generalized equation of state is shown by comparing the processing rate per iteration of the different methods. As before, the computations were made on a CRAY-YMP using the cft77 compiler. The central difference com- putations for the calorically perfect formulation and the thermally perfect formulation took $2.3078 \times 10^{-5}$ and $2.5832 \times 10^{-5}$ seconds/iteration/grid point, respectively. This represents a 11.9 percent increase in computing time to include the generalized equation of state. The 2nd order upwinding computations for the calorically perfect and thermally perfect formulations took $2.9329 \times 10^{-5}$ and $3.4121 \times 10^{-5}$ seconds/iteration/grid point, respectively. This represents a 16.3 percent increase in computing time to include the generalized equation of state.

\section{IV: Summary and Conclusions}

An efficient method to account for limited real gas effects in general Navier-Stokes computations has been presented. This method specifies the fluid pressure, temperature, laminar viscosity and thermal conductivity as explicit functions of the density and internal energy, which are simply related to the flux variables. By having an explicitly specified equation of state (EOS), no sub- iterations are required to deduce the pressure and temperature from the flux variables. The EOS approach was demonstrated by generating an equation of state for air under the assumption that it is thermally perfect, to allow for the variation of fluid specific heats with temperature. The EOS's were made available to the solver as statement functions, which allows a previously vectorized code to maintain its same basic structure and efficiency.

The flexibility of the EOS approach was demonstrated by including real gas effects in both the implicit and explicit portions of the numerical scheme. Since derivatives of the pressure with respect to the flux variables are immediately available from the EOS, they are included in the implicit portion of the scheme without any assumptions. The central difference scheme with 1st and 3rd difference dissipation originally present in the code was easily modified with the EOS formulation. A second order, TVD upwinding scheme was then added to the solver, which was also readily modified using the EOS approach. The extension to upwinding was then favorably validated against experimental data from a hypersonic corner flow experiment.

To test the installation of the real gas effects, and to compare the discretization schemes, two different flow fields were computed with and without the EOS. The first simulation was of the hypersonic flow through the corner region of two mutually perpendicular flat plates aligned with the freestream. Marked differences in wall temperatures were noted between the calorically perfect and thermally perfect formulations, as well as significant differences between the central difference 
and upwinding schemes. The second simulation was of the hypersonic flow in and around a scramjet gap seal configuration, which was represented by a corner geometry with a recessed seal, or groove, taken out along the plate intersections. Major similarities and differences between the corner and gap seal flow fields were noted, as well as differences in the shock-shock interaction phenomena predicted with the central difference and upwinding schemes. Relative efficiencies between the calorically perfect and thermally perfect gas formulations were made by comparing processing rates. These comparisons indicated that the thermally perfect EOS was approximately 12 percent slower than the calorically perfect gas mode, and that the upwinding scheme took approximately 25 percent more time than the central differencing scheme.

\section{Appendix A: Generalized Flux Jacobians}

The Jacobians of the generalized inviscid flux vectors explicitly including the pressure derivative terms from the equation of state are listed below.

Let the flux variables be

$$
q=\frac{1}{J}\left(\begin{array}{c}
\rho \\
\rho u \\
\rho v \\
\rho w \\
E_{t}
\end{array}\right)
$$

and the flux vector be

$$
F=\left(\begin{array}{c}
\rho u_{c} \\
\rho u u_{c}+\delta_{x} p \\
\rho v u_{c}+\delta_{y} p \\
\rho w u_{c}+\delta_{z} p \\
\left(E_{t}+p\right) u_{c}
\end{array}\right)
$$

The contravariant velocities are defined as

$$
u_{c}=\delta_{x} u+\delta_{y} v+\delta_{z} w
$$

where the metric terms $\delta_{x}, \delta_{y}$ and $\delta_{z}$ take the form for the $\xi$ flux

$$
\delta_{x}=\frac{\xi_{x}}{J}, \delta_{y}=\frac{\xi_{y}}{J}, \delta_{z}=\frac{\xi_{z}}{J}
$$

Then, the flux Jacobian can be formed as

$$
A=\frac{\partial F}{\partial q}
$$

so that

$$
\begin{aligned}
& A=\left(\begin{array}{cc}
0 & \delta_{x} \\
-u u_{c}+\delta_{x} p_{\rho}+\frac{\delta_{\varepsilon}}{\rho} p_{e} \phi & \delta_{x} u+u_{c}-\delta_{x} \frac{u}{\rho} p_{e} \\
-v u_{c}+\delta_{y} p_{\rho}+\frac{\delta_{x}}{\rho} p_{e} \phi & \delta_{x} v-\delta_{y} \frac{u}{\rho} p_{e} \\
-w u_{c}+\delta_{z} p_{\rho}+\frac{\delta_{\varepsilon}}{\rho} p_{e} \phi & \delta_{x} w-\delta_{z} \frac{u}{\rho} p_{e} \\
-H u_{c}+u_{c}\left(p_{\rho}+\phi \frac{p_{e}}{\rho}\right) & H \delta_{x}-u u_{c} \frac{p_{e}}{\rho}
\end{array}\right. \\
& \delta_{y} \quad \delta_{z} \\
& \delta_{y} u-\delta_{x} \frac{v}{\rho} p_{e} \quad \delta_{z} u-\delta_{x} \frac{w}{\rho} p_{e} \\
& \delta_{y} v+u_{c}-\delta_{y} \frac{v}{\rho} p_{e} \quad \delta_{z} v-\delta_{y} \frac{w}{\rho} p_{e} \\
& \delta_{y} w+u_{c}-\delta_{z} \frac{v}{\rho} p_{e} \quad u_{c}+\delta_{z} w-\delta_{z} \frac{w}{\rho} p_{e} \\
& H \delta_{y}-v u_{c} \frac{p_{e}}{\rho} \quad H \delta_{z}-w u_{c} \frac{p_{e}}{\rho} \\
& \begin{array}{c}
0 \\
\frac{\delta_{x}}{\rho} p_{e}
\end{array} \\
& \frac{\delta_{x}}{p} p_{e} \\
& \frac{\delta}{\rho} p_{e} \\
& u_{c}\left(1+\frac{p_{e}}{\rho}\right)
\end{aligned}
$$

where

$$
\begin{gathered}
U^{2}=u^{2}+v^{2}+w^{2} \\
H=\frac{E_{t}+p}{\rho} \\
\phi=-\frac{E_{t}}{\rho}+U^{2}
\end{gathered}
$$

\section{Appendix B: Frozen Air Equation of State}

The following shows the equation of state fits generated for chemically frozen air using the thermodynamic and transport data accessed by the CEC program. The supplied curve fit functions and arguments have been non-dimensionalized by the following:

$$
\begin{aligned}
& p_{\infty}=101325.0\left(\frac{N}{m^{2}}\right) \\
& T_{\infty}=300.0(\text { Kelvin })
\end{aligned}
$$




$$
\begin{aligned}
\rho_{\infty} & =1.17196272\left(\frac{K g}{m^{3}}\right) \\
e_{\infty} & =8.64571924 \times 10^{4}\left(\frac{m^{2}}{s^{2}}\right) \\
\mu_{\infty} & =180.16133(\mu P) \\
\lambda_{\infty} & =12.619813\left(\frac{\mu W}{c m K}\right)
\end{aligned}
$$

The EOS functions for pressure, temperature, laminar viscosity and thermal conductivity are

$$
\begin{aligned}
p(\rho, e)= & a_{1} e+a_{2} \rho e+a_{3}+a_{4} \rho e^{2}+a_{5} \rho e^{3}+\quad(B .2 . a) \\
& a_{6} \rho e^{4}+a_{7} \rho^{2} e^{3}+a_{8} \rho^{2} e^{4}+a_{9} \rho e^{5}+ \\
& a_{10} \rho^{2} e^{5}+a_{11} \rho^{2} e^{6}+a_{12} \rho e^{6}+a_{13} \rho^{2} e^{7}+a_{14} \rho e^{7} \\
\frac{\partial p}{\partial \rho}= & a_{2} e+a_{4} e^{2}+a_{5} e^{3}+a_{6} e^{4}+2 a_{7} \rho e^{3}+\quad(B .2 . b) \\
& 2 a_{8} \rho e^{4}+a_{9} e^{5}+2 a_{10} \rho e^{5}+2 a_{11} \rho e^{6}+ \\
& a_{12} e^{6}+2 a_{13} \rho e^{7}+a_{14} e^{7} \\
\frac{\partial p}{\partial e}= & a_{1}+a_{2} \rho+2 a_{4} \rho e+3 a_{5} \rho e^{2}+4 a_{6} \rho e^{3}+(B .2 . c) \\
& 3 a_{7} \rho^{2} e^{2}+4 a_{8} \rho^{2} e^{3}+5 a_{9} \rho e^{4}+5 a_{10} \rho^{2} e^{4}+ \\
& 6 a_{11} \rho^{2} e^{5}+6 a_{12} \rho e^{5}+7 a_{13} \rho^{2} e^{6}+7 a_{14} \rho e^{6}
\end{aligned}
$$

$$
\begin{aligned}
T(\rho, e)= & b_{1} e / \rho+b_{2} e+b_{3} / \rho+b_{4} e^{2}+b_{5} e^{3}+b_{6} e^{4}(B .3 . a) \\
& +b_{7} \rho e^{3}+b_{8} \rho e^{4}+b_{9} e^{5}+b_{10} \rho e^{5}+b_{11} \rho e^{6}+ \\
& b_{12} e^{6}+b_{13} \rho e^{7}+b_{14} e^{7} \\
\frac{\partial T}{\partial \rho}= & -b_{1} e / \rho^{2}-b_{3} / \rho^{2}+b_{7} e^{3}+b_{8} e^{4}+b_{10} e^{5}(B .3 . b) \\
& +b_{11} e^{6}+b_{13} e^{7} \\
\frac{\partial T}{\partial e}= & b_{1} / \rho+b_{2}+2 b_{4} e+3 b_{5} e^{2}+4 b_{6} e^{3}+ \\
& 3 b_{7} \rho e^{2}+4 b_{8} \rho e^{3}+5 b_{9} e^{4}+5 b_{10} \rho e^{4}+ \\
& 6 b_{11} \rho e^{5}+6 b_{12} e^{5}+7 b_{13} \rho e^{6}+7 b_{14} e^{6} \\
\mu(T)= & c_{1}+c_{2} T^{\frac{1}{2}}+c_{3} T+c_{4} T^{\frac{3}{2}}+ \\
& c_{5} T^{2}+c_{6} T^{\frac{5}{2}}+c_{7} T^{3} \\
\lambda(T)= & d_{1}+d_{2} T^{\frac{1}{2}}+d_{3} T+d_{4} T^{\frac{3}{2}}+ \\
& d_{5} T^{2}+d_{6} T^{\frac{5}{2}}+d_{7} T^{3}
\end{aligned}
$$

The EOS coefficients $a_{n}, b_{n}, c_{n}$ and $d_{n}$ are tabulated below.

$$
\begin{array}{ccc}
\mathrm{n} & a_{n} & b_{n} \\
1 & -1.64184011 \times 10^{-08} & -4.66744619 \times 10^{-11} \\
2 & +4.25555113 \times 10^{-01} & +4.11592537 \times 10^{-01} \\
3 & +2.87728820 \times 10^{-06} & +9.27096790 \times 10^{-09} \\
4 & -6.01056836 \times 10^{-03} & -4.76679827 \times 10^{-03} \\
5 & +1.16745176 \times 10^{-04} & +8.29280665 \times 10^{-05} \\
6 & -1.18673130 \times 10^{-06} & -7.78921374 \times 10^{-07} \\
7 & +1.68428888 \times 10^{-07} & +1.35065409 \times 10^{-08} \\
8 & -4.49056339 \times 10^{-09} & -3.13912759 \times 10^{-10} \\
9 & +6.28302532 \times 10^{-09} & +3.84665192 \times 10^{-09} \\
10 & +4.16636684 \times 10^{-11} & +2.65689892 \times 10^{-12} \\
11 & -1.62288924 \times 10^{-13} & -9.73342645 \times 10^{-15} \\
12 & -1.66714128 \times 10^{-11} & -9.60514023 \times 10^{-12} \\
13 & +2.26513998 \times 10^{-16} & +1.30418711 \times 10^{-17} \\
14 & +1.75501828 \times 10^{-14} & +9.61090540 \times 10^{-15} \\
& &
\end{array}
$$

\section{References}

(1) Pulliam, T.H., and Steger, J.L., "Implicit FiniteDifference Simulations of Three Dimensional Compressible Flow," AIAA Journal, Vol. 18, No. 2, Feb. 1980, pp. 159-167.

(2) Beam, R. and Warming, R.F., "An Implicit FiniteDifference Algorithm for Hyperbolic Systems in Conservation-Law Form," Journal of Computational Physics, Vol. 22, No. 1, Sept. 1976, pp. $87-110$.

(3) Pulliam, T.H., "Euler and Thin Layer NavierStokes Codes: ARC2D,ARC3D," Notes for Computational Fluid Dynamics User's Workshop, The 
University of Tennessee Space Institute, Tullahoma, Tennessee, (UTSI Publication E02-4005023-84), March 12-16, 1984, pp. 15.1-15.85.

(4) Cooper, G.K., "The PARC Code: Theory and Usage," AEDC-TR- 87-24, October, 1987.

(5) Coirier, W.J., "High Speed Corner and Gap-Seal Calculations using an LU Scheme," AIAA paper 89-2669, July, 1989.

(6) Jameson, A. and Turkel, E., "Implicit Schemes and LU Decompositions," Mathematics of Computation, Vol. 37, No. 156, 1981, pp. 385-397.

(7) Jameson, A. and Yoon, S., "LU Implicit Schemes with Multiple Grids for the Euler Equations," AIAA paper 86-0105, January, 1986.

(8) Shuen, J.S. and Yoon, S., "Numerical Study of Chemically Reacting Flows Using an LU Scheme," AIAA paper 88-0436, January, 1988.

(9) Yu, S.T., Tsai, Y.L. and Shuen, J.S., "ThreeDimensional Calculation of Supersonic Reacting Flows using an LU Scheme," AIAA paper 89-0391, January, 1989.

(10) Liou, M.S. and Hsu, A. T., "A Time Accurate Finite Volume High Resolution Scheme for Three Dimensional Navier-Stokes Equations,", AIAA paper 89-1994, June, 1989.

(11) Van Leer, B., "Flux-Vector Splitting for the Euler Equations," Lecture Notes in Physics, Vol. 170, 1982, Springer-Verlag, Berlin.

(12) Mani,M. and Tiwari, S. N., "Investigation of TwoDimensional Chemically Reacting and Radiating Supersonic Channel Flows," AIAA paper 88-0462, January, 1988.

(13) Yu, S.T., McBride, B.J., Hsieh, K.C., and Shuen, J.S., "Numerical Simulation of Hypersonic Inlet Flows with Equilibrium or Finite Rate Chemistry,", AIAA paper 88-0273, January 1988.

(14) Shuen, J.S. and Liou, M.S., "Flux Splitting Algorithms for Two-Dimensional Viscous Flows with Finite-Rate Chemistry," AIA A paper 89-0388, January, 1989.

(15) Liou, M.S., Van Leer, B. and Shuen, J.S., "Splitting of Inviscid Fluxes for Real Gases.", to appear in Journal of Computational Physics .

(16) Liou, M.F., "Three Dimensional PNS Solutions of Hypersonic Internal Flows with Equilibrium Chemistry,", AIAA paper 89-0002, January, 1989.

(17) Gordon, S. and McBride, B.J., "Computer Program for the Calculation of Complex Equilibrium Compositions, Rocket Performance, Incident and Reflected Shocks, and Chapman-Jouget Detonations,", NASA SP-273 Interim Revision, 1976.

(18) Gordon, S., McBride, B.J. and Zeleznik, F.J., "Computer Program for Calculation of Complex Chemical Equilibrium Compostions and Applica- tions Supplement I - Transport Properties," NASA TM-86885, October, 1984.

(19) Yu, S.T. and McBride, B.J., "Numerical Simulation of Hypersonic Inlet Flows with Equilibrium or Finite Rate Chemistry,", AIAA paper 88-0273, January, 1988.

(20) Natl. Bur. Stand. (U.S.) Circ. 564, 1955.

(21) Jameson, A. Schmidt, W. and Turkel, E., "Numerical Solutions of the Euler Equations by Finite Volume Methods using Runge-Kutta Time Stepping Schemes," AIAA Paper 81-1259, 1981.

(22) Yoon, S. and Jameson, A., "Lower-Upper Implicit Scheme for High-Speed Inlet Analysis," AIAA Journal, Vol. 25, Aug. 1987, pp 1052-1053.

(23) Yoon, S. and Jameson, A., "An LU-SSOR Scheme for the Euler and Navier-Stokes Equations," AIAA paper 87-0600, January, 1987.

(24) Park, C. and Yoon, S., "Calculation of Real-Gas Effects on Blunt-Body Trim Angles," AIAA paper 89-0685, Jan. 1989.

(25) Yoon, S. and Kwak, D., "Artificial Dissipation Models for Hypersonic External Flows," AIAA paper 88-3708, July, 1988.

(26) Steinetz, B.M., DellaCorte, C. and Sirocky, P., "On the Development of Hypersonic Engine Seals" NASA TP-2854, December, 1988.

(27) Cresci, R.J., "Hypersonic Flow Along Two Intersecting Planes," AFOSR 66-0500, March 1966.

(28) Kandil, O.A. and Chuang, A.H., "Influence of Numerical Dissipation on Computational Euler Equations for Vortex-Dominated Flows," AIAA Journal , Vol. 25, No. 11, pp.1426-1434, November, 1987. 




Figure 1. Equation of State Comparison: Pressure

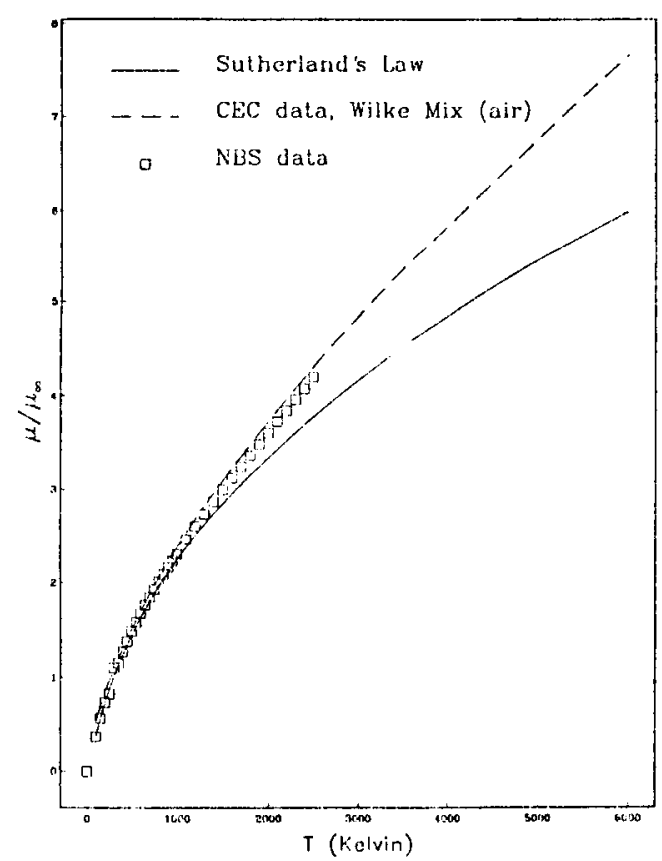

Figure 3. Equation of State Comparison: Laminar Viscosity

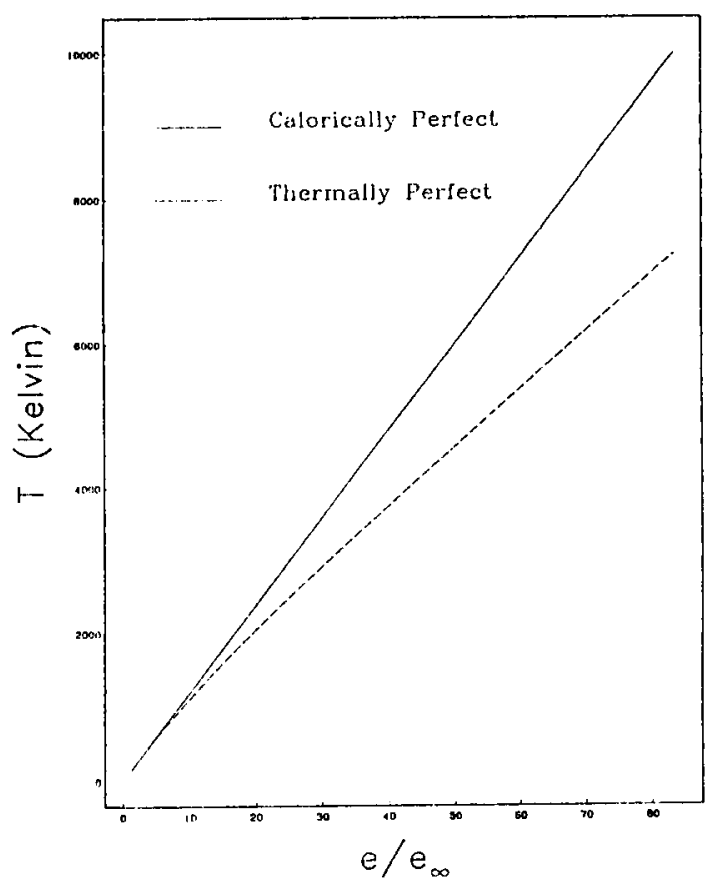

Figure 2. Equation of State Comparison: Temperature



Figure 4. Equation of Statc Comparison: Thermal Conductivity 


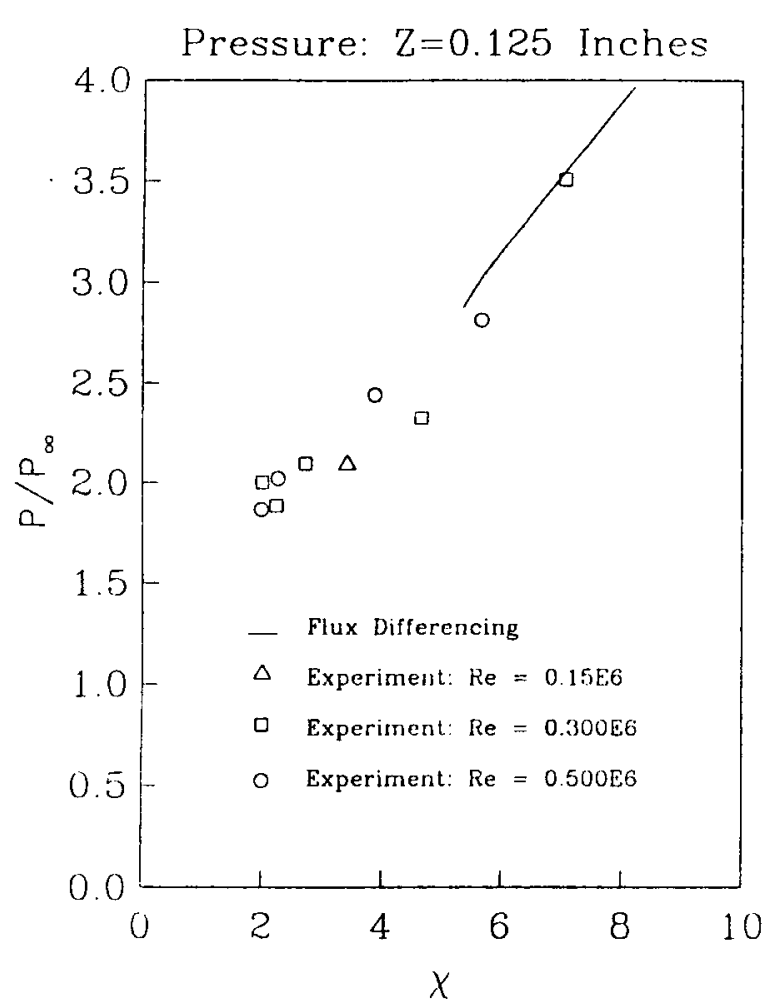

Figure 5. Corner Validation Case: Pressure

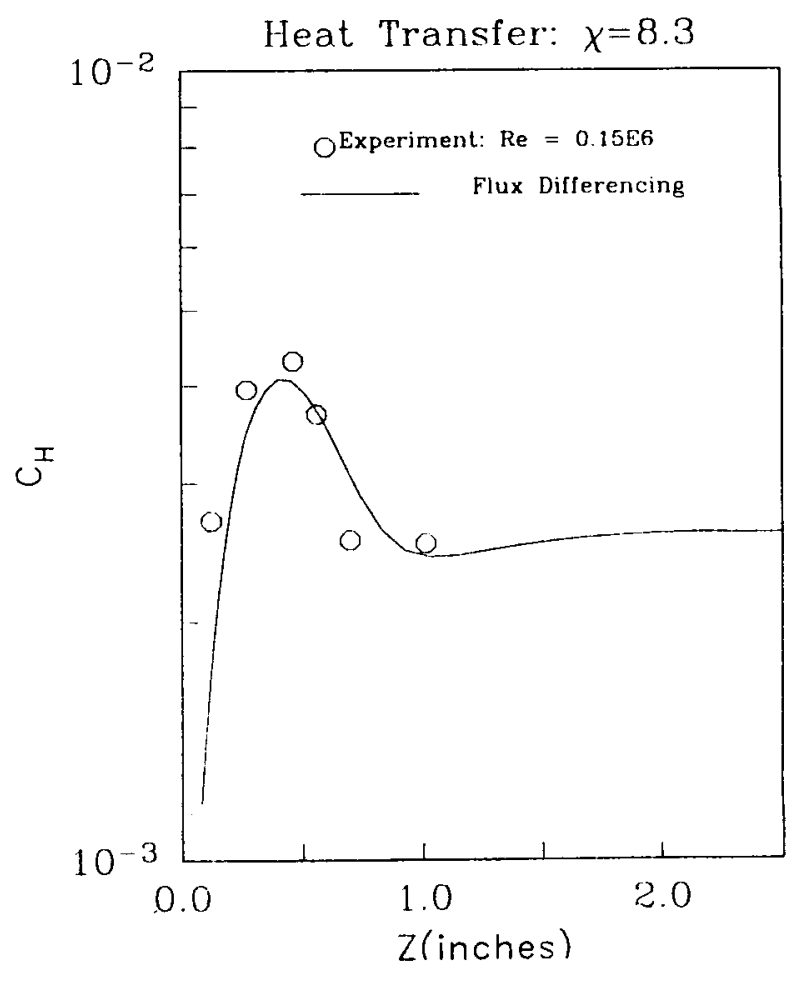

Figure 6. Corner Validation Case: Heat Transfer

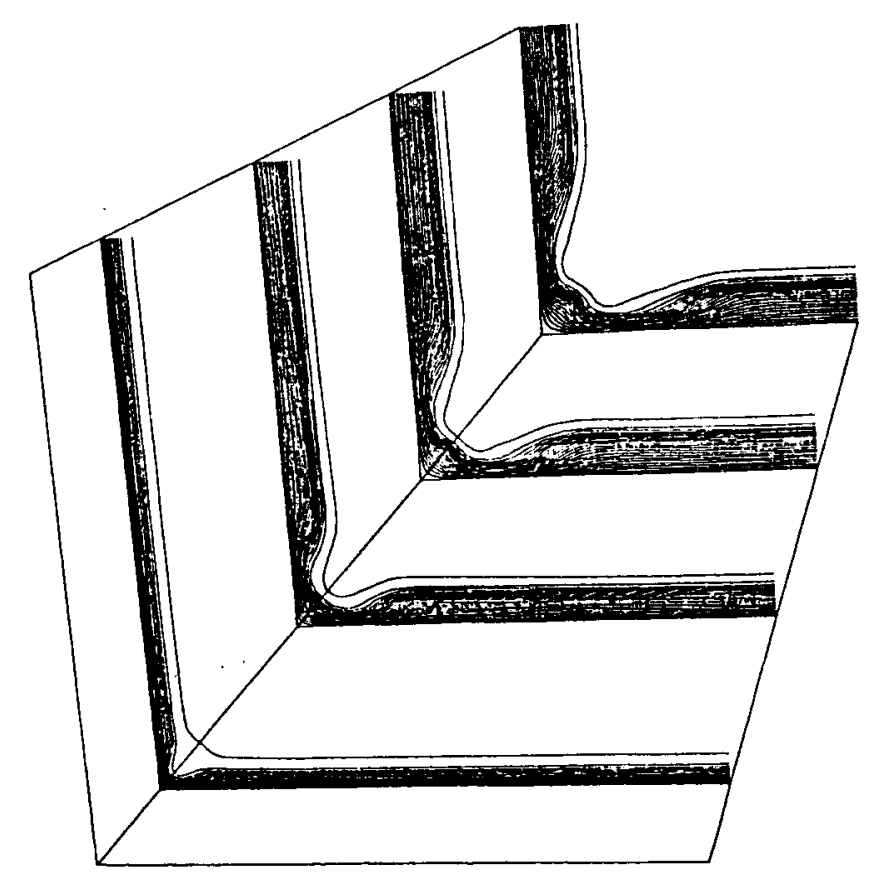

Figure 7. Axial Velocity Contours: Corner Case 


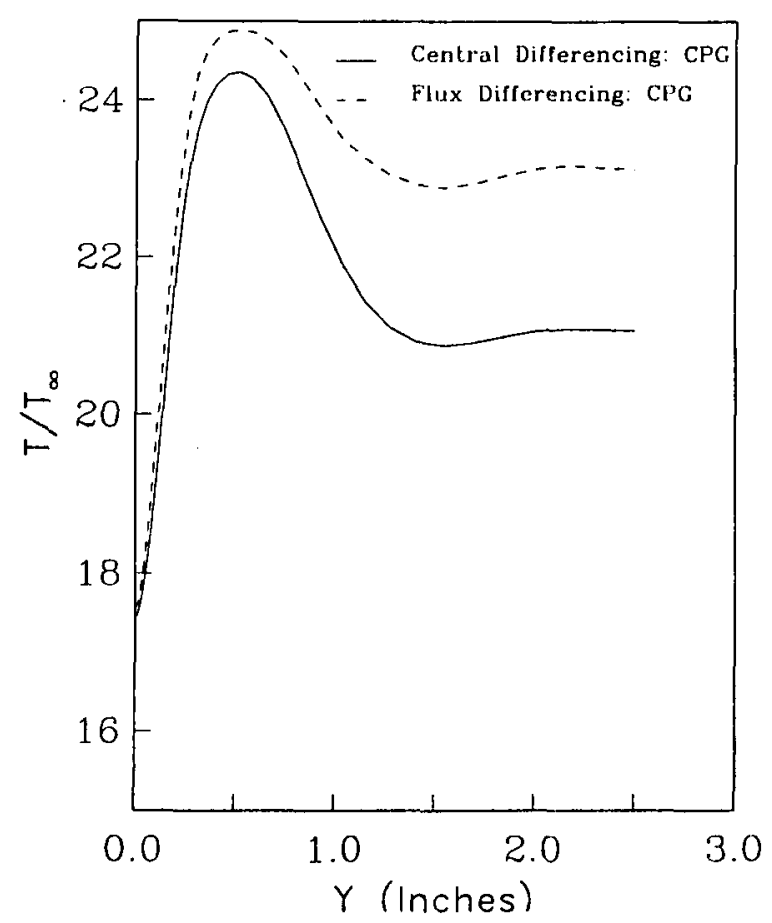

Figure 8. Spanwise Wall Temperature Variation: Corner Case

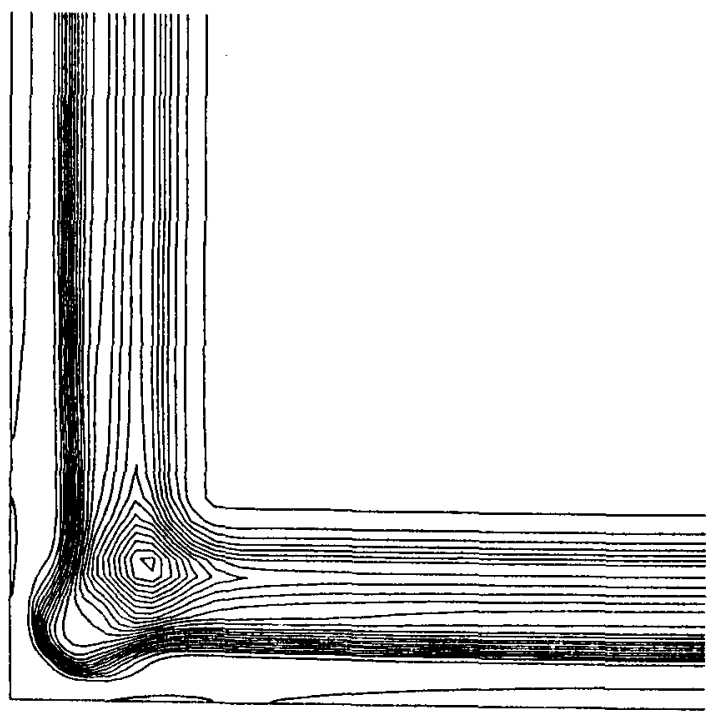

Figure 10.a Density Contours, Central Differencing: Corner Case

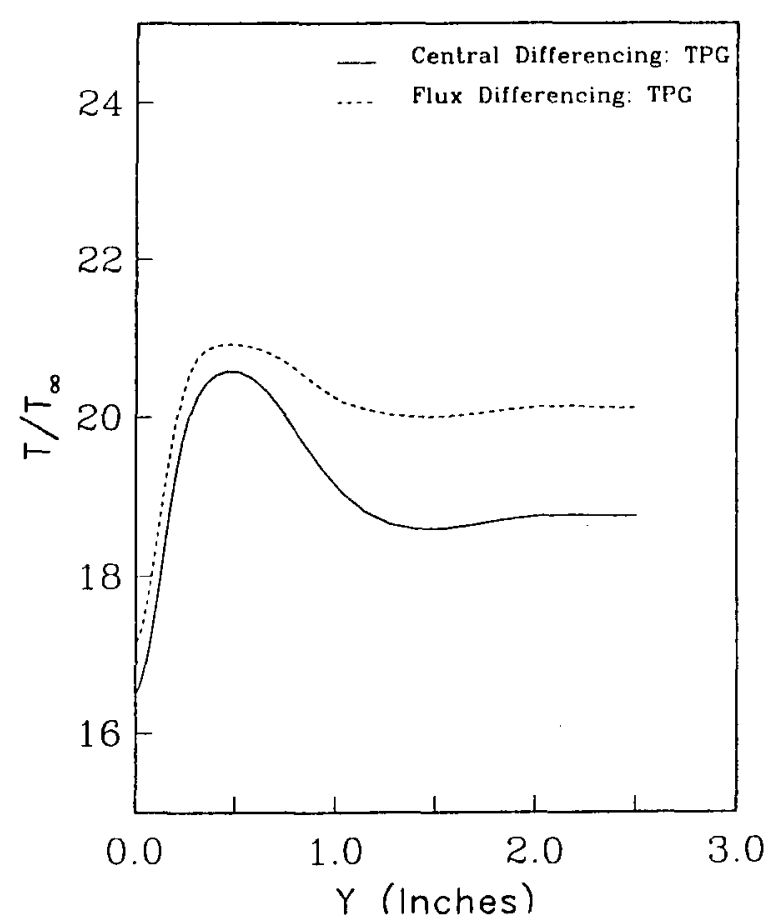

Figure 9. Spanwise Wall Temperature Variation: Corner Case

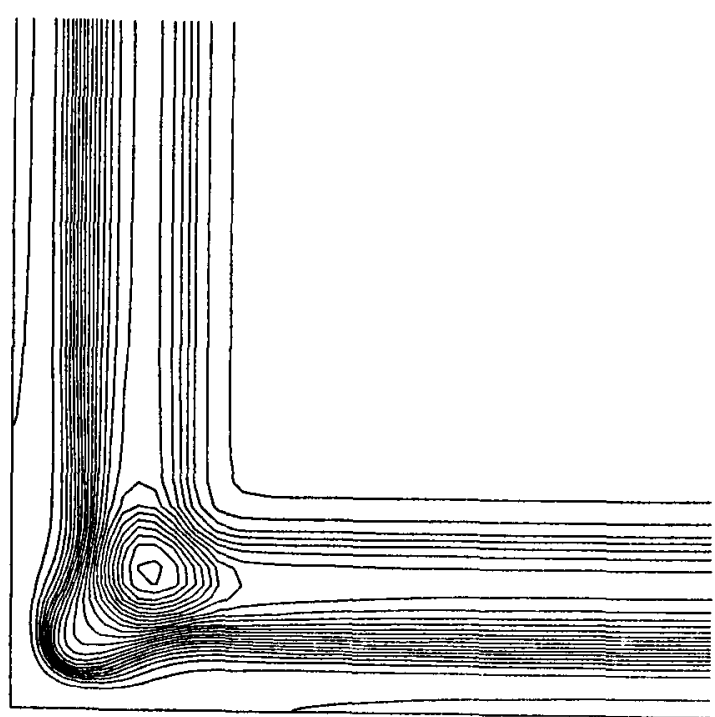

Figure 10.b Density Contours, Flux Differencing: Corner Case 


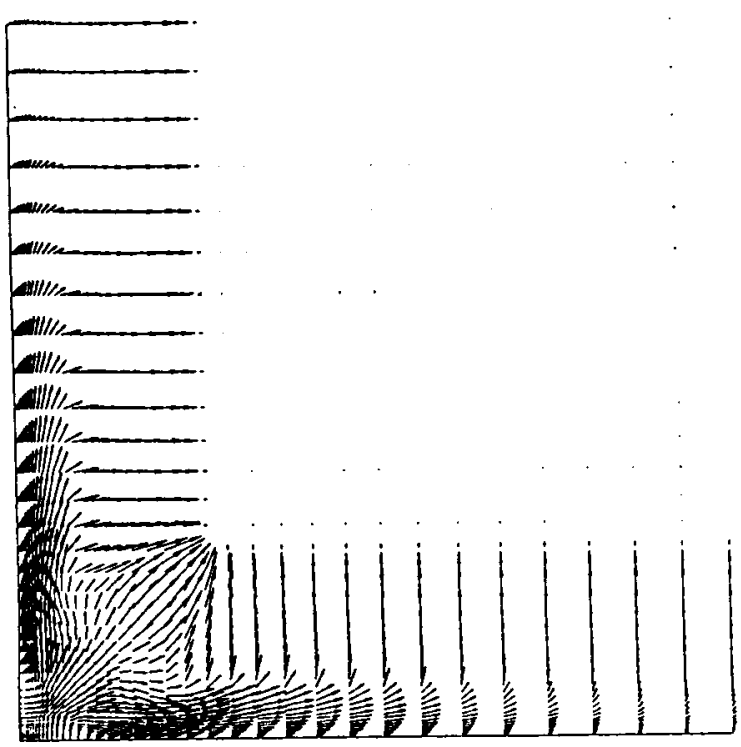

Figure 11.a Cross Flow Velocity Vectors, Central Differencing: Corner Case

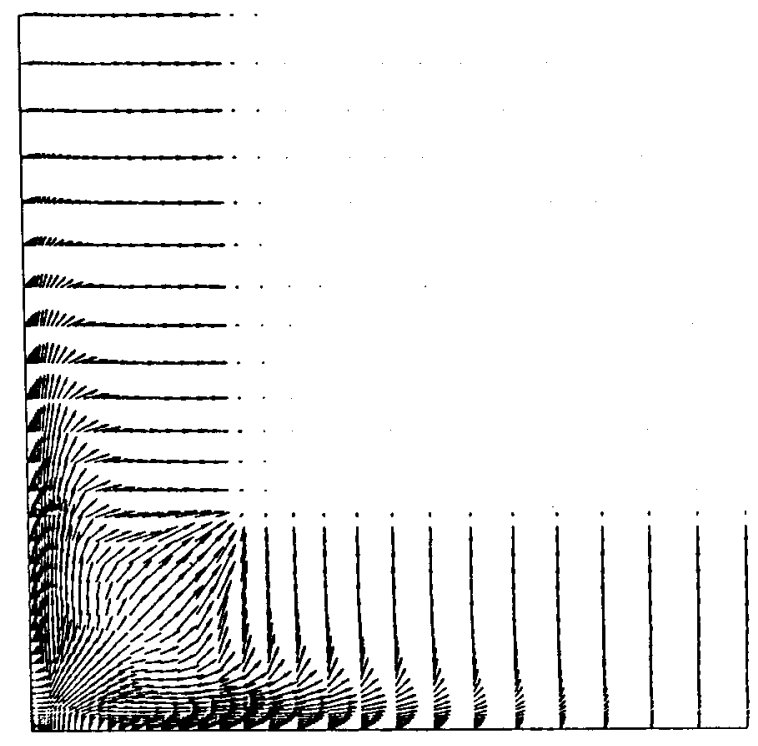

Figure 11.b Cross Flow Velocity Vectors, Flux Differencing: Corner Case

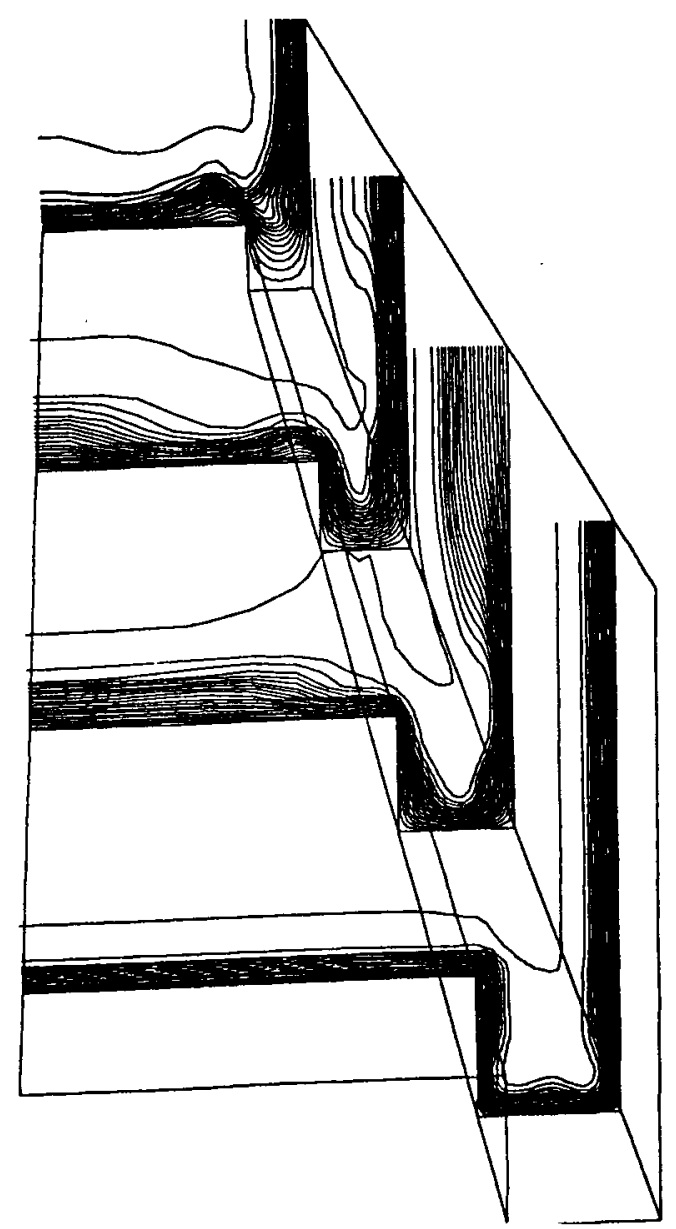

Figure 12. Axial Velocity Contours: Gap Seal Case 


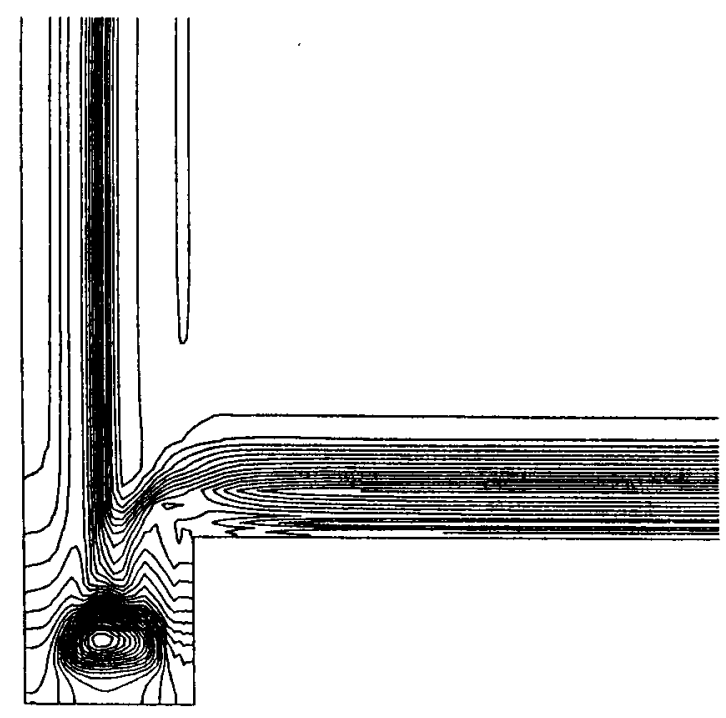

Figure 13.a Pressure Contours, Central Differencing: Gap Seal Case

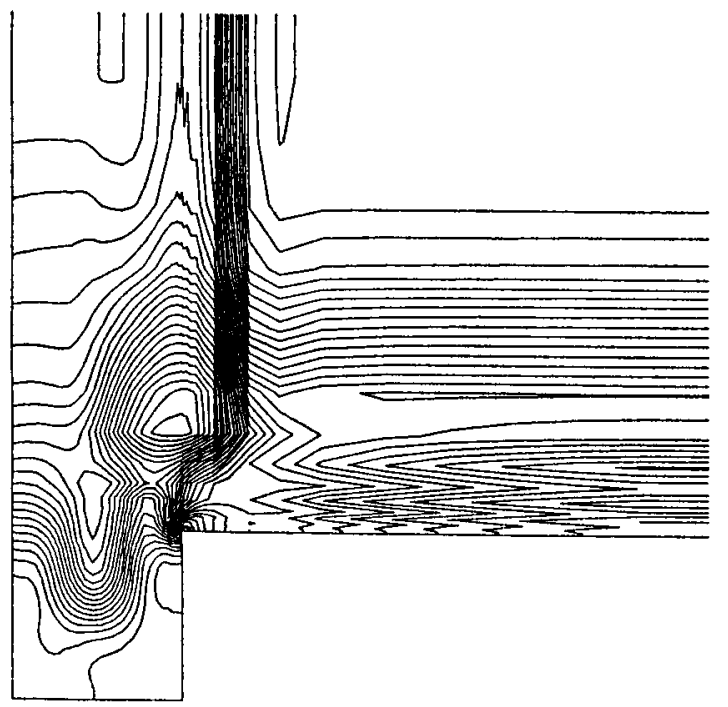

Figure 14.a Pressure Contours, Central Differencing: Gap Seal Case

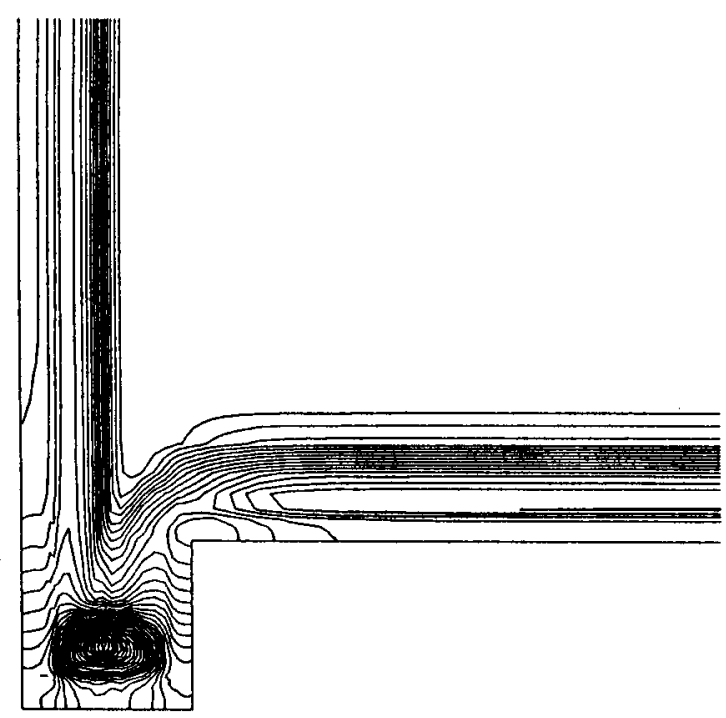

Figure 13.b Pressure Contours, Flux Differencing: Gap Seal Case

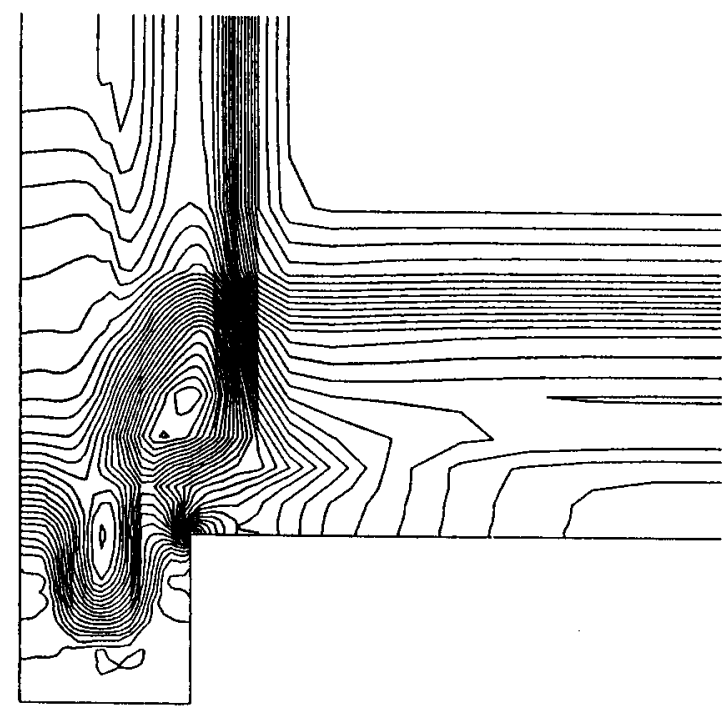

Figure 14.b Pressure Contours, Flux Differencing: Gap Seal Case 


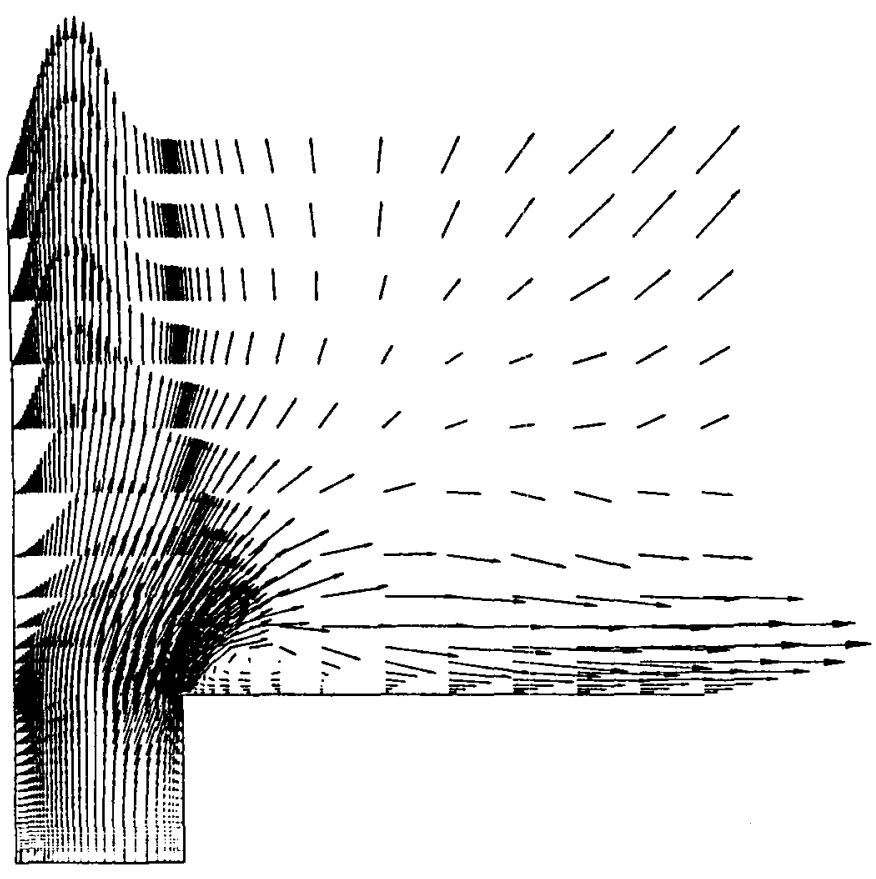

Figure 15.a Cross Flow Velocity Vectors, Central Differencing: Gap Seal Case

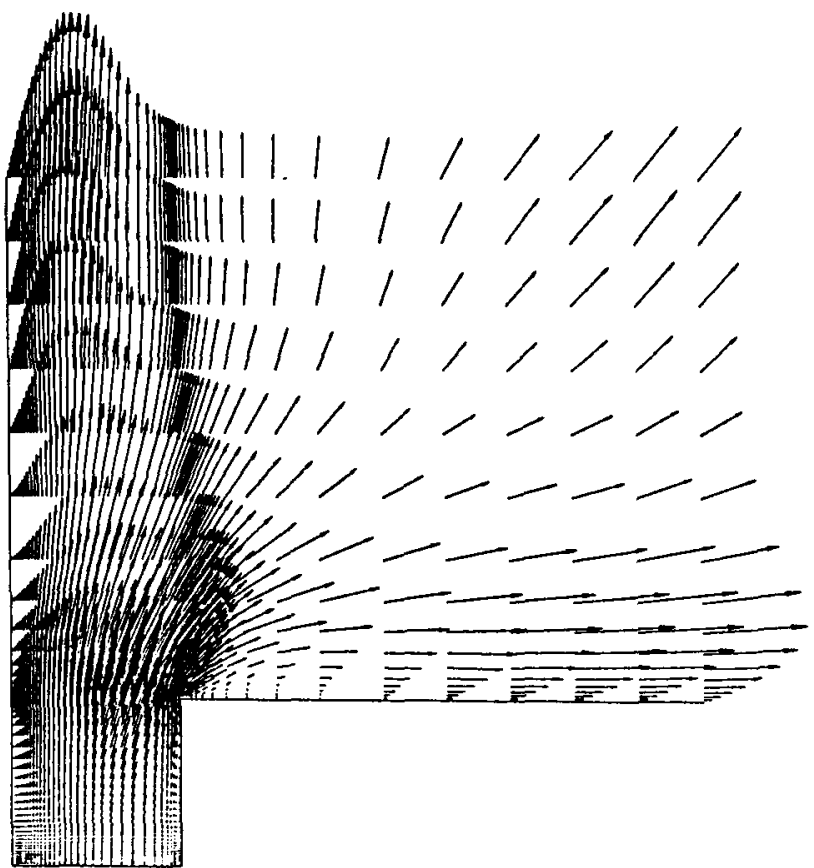

Figure 15.b Cross Flow Velocity Vectors, Flux Differencing: Gap Seal Case

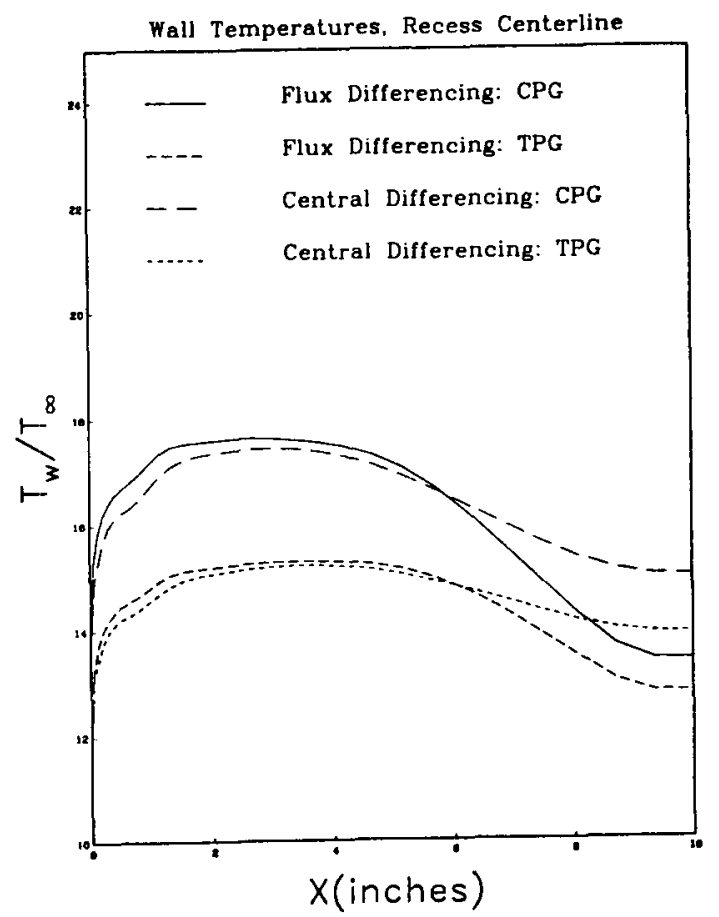

Figure 16. Recess Centerline Wall Temperatures 


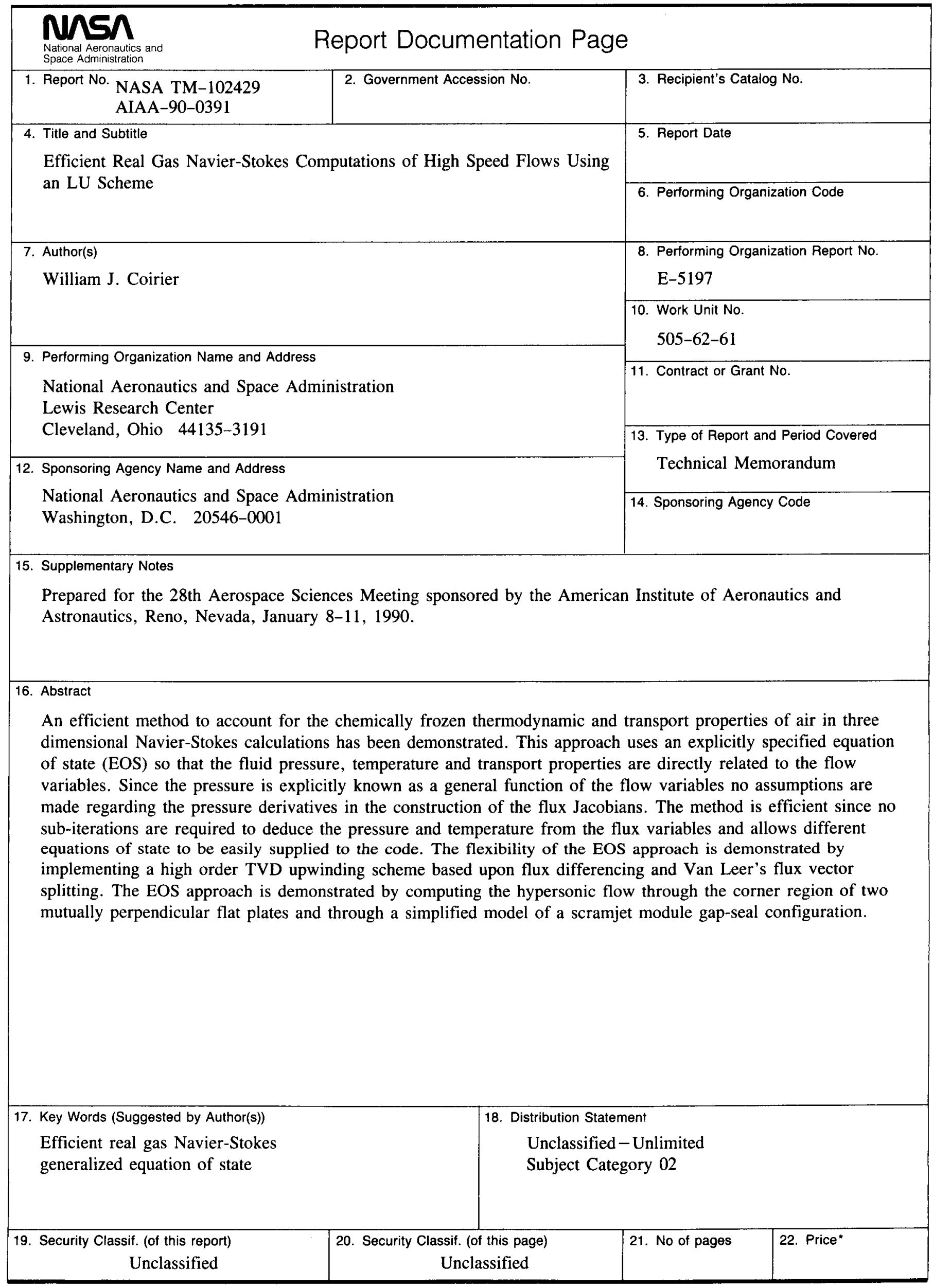

